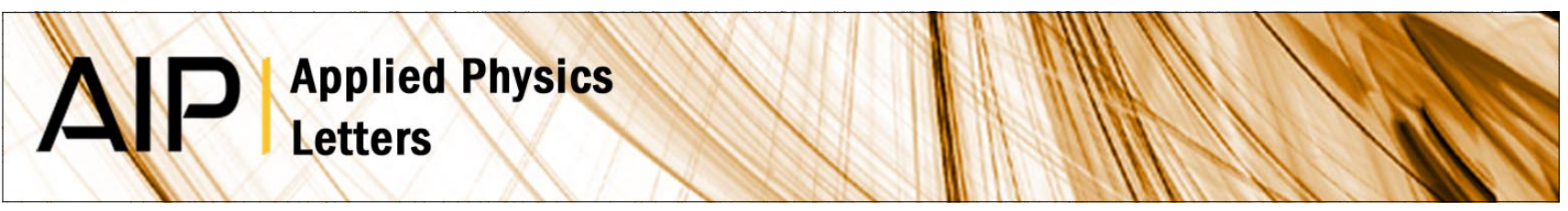

\title{
Dual integrated actuators for extended range high speed atomic force microscopy
}

T. Sulchek, S. C. Minne, J. D. Adams, D. A. Fletcher, A. Atalar et al.

Citation: Appl. Phys. Lett. 75, 1637 (1999); doi: 10.1063/1.124779

View online: http://dx.doi.org/10.1063/1.124779

View Table of Contents: http://apl.aip.org/resource/1/APPLAB/v75/i11

Published by the American Institute of Physics.

\section{Additional information on Appl. Phys. Lett.}

Journal Homepage: http://apl.aip.org/

Journal Information: http://apl.aip.org/about/about_the_journal

Top downloads: http://apl.aip.org/features/most_downloaded

Information for Authors: http://apl.aip.org/authors

\section{ADVERTISEMENT}

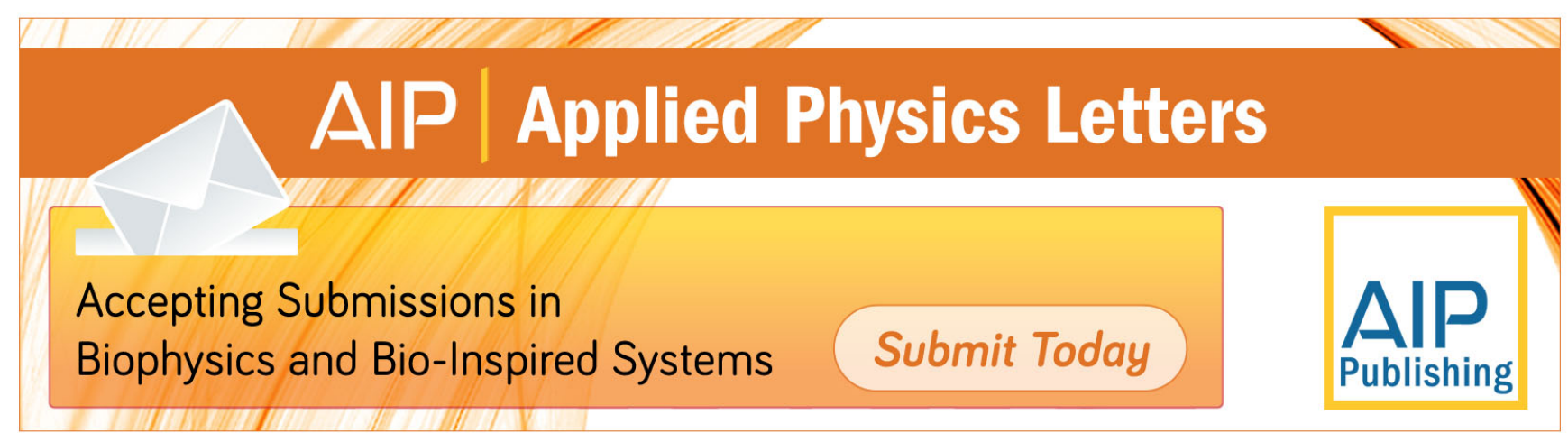




\title{
Dual integrated actuators for extended range high speed atomic force microscopy
}

\author{
T. Sulchek, ${ }^{\text {a) }}$ S. C. Minne, J. D. Adams, D. A. Fletcher, A. Atalar, and C. F. Quate \\ E. L. Ginzton Laboratory, Stanford University, Stanford, California 94305-4085 \\ D. M. Adderton \\ NanoDevices Inc., 3887 State Street \#107, Santa Barbara, California 93105
}

(Received 1 June 1999; accepted for publication 19 July 1999)

\begin{abstract}
A flexible system for increasing the throughput of the atomic force microscope without sacrificing imaging range is presented. The system is based on a nested feedback loop which controls a micromachined cantilever that contains both an integrated piezoelectric actuator and an integrated thermal actuator. This combination enables high speed imaging $(2 \mathrm{~mm} / \mathrm{s})$ over an extended range by utilizing the piezoelectric actuator's high bandwidth $(15 \mathrm{kHz})$ and thermal actuator's large response $(300 \mathrm{~nm} / \mathrm{V})$. A constant force image, where the sample topography exceeds the range of the piezoelectric actuator alone, is presented. It has also been demonstrated that the deflection response of the thermal actuator can be linearized and controlled with an integrated diode. (C) 1999 American Institute of Physics. [S0003-6951(99)05237-7]
\end{abstract}

The atomic force microscope (AFM) has become an essential instrument for visualizing, monitoring, and characterizing surfaces. Since its introduction in 1986 there has been a steady stream of innovations which have increased the sensitivity and functionality of the instrument. Current state of the art for AFMs can now map topography, friction, electric, and magnetic fields, temperature, and more. When imaging, vertical resolutions of less than $1 \mathrm{~A}$ are routinely achieved. However, for most AFMs, the throughput, or the time it takes to acquire an image, has not improved. There have been many demonstrations of systems which enhance the bandwidth of the microscope, but these speed enhancements come at the expense of the microscope's vertical range. By simultaneously integrating a large displacement thermal actuator with a high speed piezoelectric actuator, we have shown that the speed of the AFM can be improved by over an order of magnitude without sacrificing the instrument's vertical range.

In constant force AFM imaging, the speed of the microscope is generally limited by the mechanical response of the piezotube. For a typical 2 in. piezotube scanner, this bandwidth is roughly $600 \mathrm{~Hz}$. Previous work on enhancing the speed of the AFM has focused on this bandwidth limitation. The primary method for addressing this problem has been to replace the primary feedback actuator with a faster one. This has been successfully accomplished by depositing piezoelectric films onto the cantilever. Several groups have deflected cantilevers using this technique. ${ }^{1-3}$ High speed imaging using devices with integrated actuators has been shown with piezoresistive sensors ${ }^{4,5}$ and optical lever sensors. ${ }^{6}$

In previous work ${ }^{6}$ the full capabilities of the micromachined device were used in order to speed up the AFM. However, the throughput enhancements of this technique are not scaleable. The speed of the AFM in contact mode is limited by the resonance of the feedback actuator. For an actuator integrated onto a cantilever, the resonant frequency

a)Electronic mail: sulchek@stanford.edu of the cantilever will scale as $t / L^{2}$ (where $t$ is the thickness of the cantilever and $L$ is the length of the cantilever). Unfortunately, the maximum piezoelectric induced deflection of the cantilever ${ }^{7}$ scales as approximately $L^{2} / t$. With this approach, further throughput increases in AFM imaging will come at the expense of the AFMs imaging range.

The work presented here picks up where the work in Ref. 7 leaves off. It should be noted that the image presented in this letter is of the same sample that was presented in that reference. Here we show that with the dual actuator approach, we can reduce the voltage applied to the piezoelectric actuator by a factor of two without sacrificing speed. This indicates that, with the appropriate sensor, the total microscope $z$ range could be increased without loss of speed, or, if the cantilever's dimensions were appropriately redesigned, the total image speed could be increased without loss of range in the $z$ dimension. It is possible to scale this tradeoff further as only a small fraction of the thermal actuator's range is used. (A cantilever deflection of several microns will move the beam in the optical lever system off the photodetector, and thus will require that the traditional split photodiode detector be replaced with a linear graded photodetector, or the interdigital detection system. ${ }^{8}$ ) It should be noted that the maximum slew rate in $z$ is only available over the $z$ range of the fast (piezoelectric) actuator. Thus, for example, the dual actuator will not accurately track a step greater than the range of the fast actuator. We, however, often encounter samples with features that are microns in height with slow variation in the lateral plane, i.e., low spatial frequency. The fine features with high spatial frequencies have small (nm) amplitudes. The thermal actuator with its slow response is ideal for this situation since it can accommodate a tip displacement of several microns. This large response was attained without optimization of the thermal actuator. We expect a larger bandwidth without loss of response by placing the heater in the bimorph region itself.

Figure 1 shows a schematic diagram for implementing both the thermal and piezoelectric actuator where the deflec- 


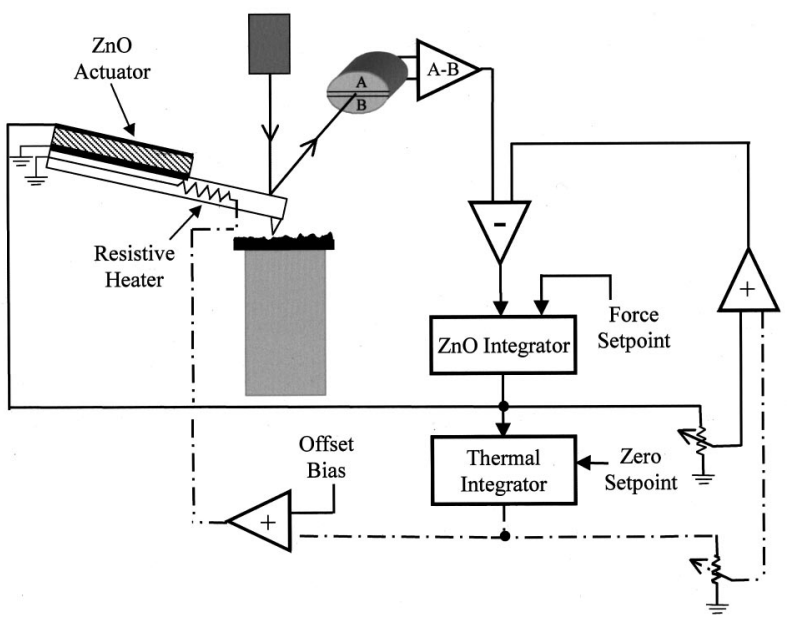

FIG. 1. A schematic diagram for an AFM with integrated piezoelectric and integrated thermal actuators operating in nested feedback. The system includes two linear corrections; one to compensate for each actuator's induced angle offset. A dc bias is applied to the thermal actuator signal to ensure its value always remains positive.

tion is monitored with the optical lever. The description and fabrication process for the cantilever used in this work has been described elsewhere. ${ }^{9}$ The design presented in Fig. 1 uses a nested feedback configuration which requires no filters. During operation, the fast $\mathrm{ZnO}$ actuator nulls the topographic signal (just as in a standard AFM), but the slower, thermal actuator responds to large scale features in topography in the attempt to keep the fast actuator's signal zero. This configuration allows the high speed actuator to stay centered within its range so that it can respond with its full displacement. The thermal actuator, on the other the hand, will maintain constant force on the slowly varying features. This is evident in the transfer function of the $\mathrm{ZnO}$ actuator in closed loop dual feedback operation of Fig. 2. The response of the $\mathrm{ZnO}$ at dc goes to zero, which means that the $\mathrm{ZnO}$ will not monitor the average sample position but only the high frequency data (up to $15 \mathrm{kHz}$ using a $45^{\circ}$ phase margin).

Referring back to Fig. 1, it should be pointed out that both the $\mathrm{ZnO}$ actuator's response and the thermal actuator's response must be scaled and summed with the topographic signal. This method for correcting cantilever movement for optical detection was first reported by Manalis et al. ${ }^{6}$ To summarize, the optical lever measures the angle of the cantilever. In microscopes which either move the sample or the entire cantilever as a unit, this angle only changes when a force is acting on the tip. However, when using an integrated actuator, motion in the vertical direction is achieved by changing the angle of the cantilever itself. Thus, even if constant force is maintained by the integrated actuator, a nonzero optical lever signal will result. To eliminate the actuator induced angle, we subtract an appropriately scaled signal from the output of the photodiode. In order to calibrate the correction circuitry, the actuators are separately modulated while the tip is free. For each actuator, the correction circuitry is adjusted until the free air deflection is nulled. Constant force has been verified by Manalis ${ }^{6}$ through independent measurement of strain with a piezoresistive sensor, and can be ensured while imaging by observing that the error signal is less than one percent of the imaging signal. (a)

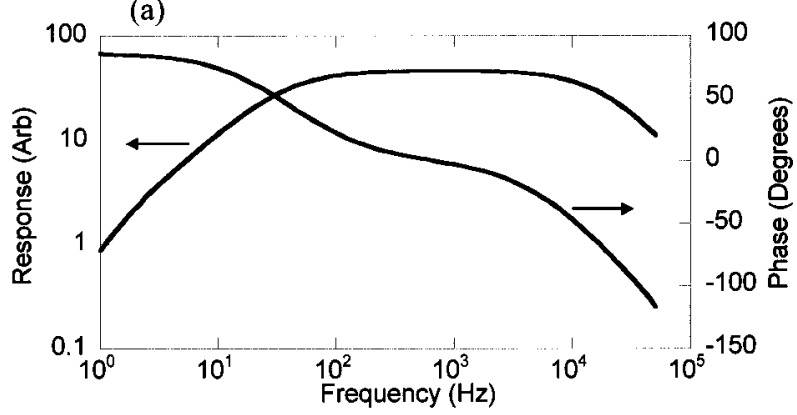

(b)

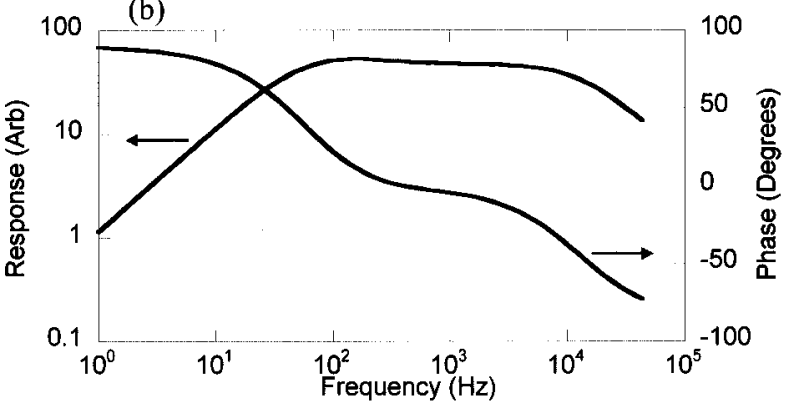

FIG. 2. (a) Measured and (b) simulated amplitude and phase data for the $\mathrm{ZnO}$ actuator in closed loop dual feedback operation. With a margin in phase of $45^{\circ}$, the imaging bandwidth is $15 \mathrm{kHz}$. The drop-off below $60 \mathrm{~Hz}$ is due to the response of the thermal actuator.

In the cantilever used for this experiment, the resistive heater for the thermal actuation consists of a $p$-type region implanted into $n$-type silicon; the resistance of the path is roughly $3 \mathrm{k} \Omega$. Heat is generated in the cantilever by Joule heating in the resistor, so that the different thermal expansion coefficients between silicon and $\mathrm{ZnO}$ create a stress throughout the bimorph which is relieved by bending.

For small applied voltages, the power follows a $V^{2} / R$ relation. Vertical deflection of a cantilever bimorph is proportional to temperature, which is proportional to power, ${ }^{10}$ and thus for low bias deflection varies with $V^{2}$. However, when the $p-n$ junction isolation is biased beyond $12 \mathrm{~V}$, we encounter $p-n$ junction breakdown. Breakdown is seen by a
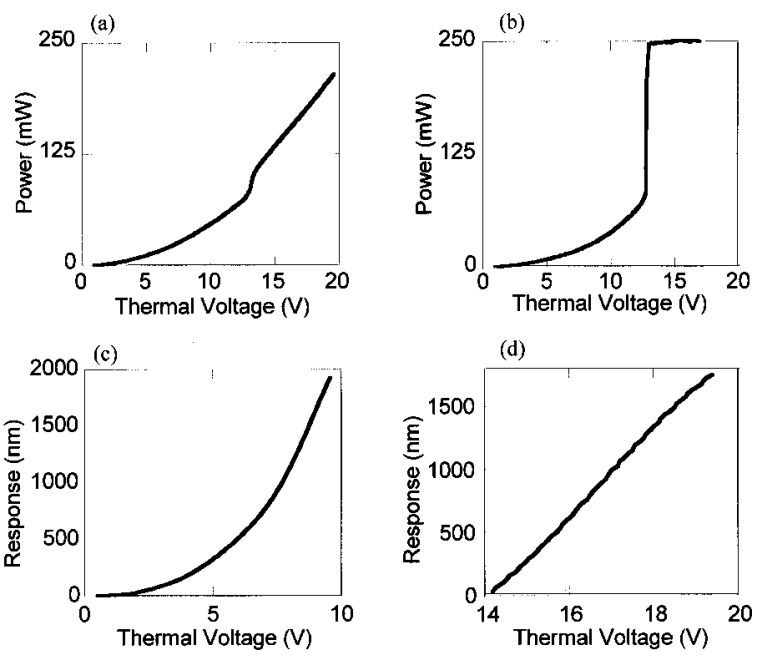

FIG. 3. Thermal actuator dissipated power vs voltage ramped over time for (a) the resistive heater in series with a $3 \mathrm{k} \Omega$ external resistor and (b) the resistive heater in series with a $30 \Omega$ external resistor. Increasing the size of the series resistor will control and linearize the thermal actuator's deflection response in the breakdown region. Measured deflection of the cantilever is shown in the parabolic region (c) and the linear breakdown region (d). 

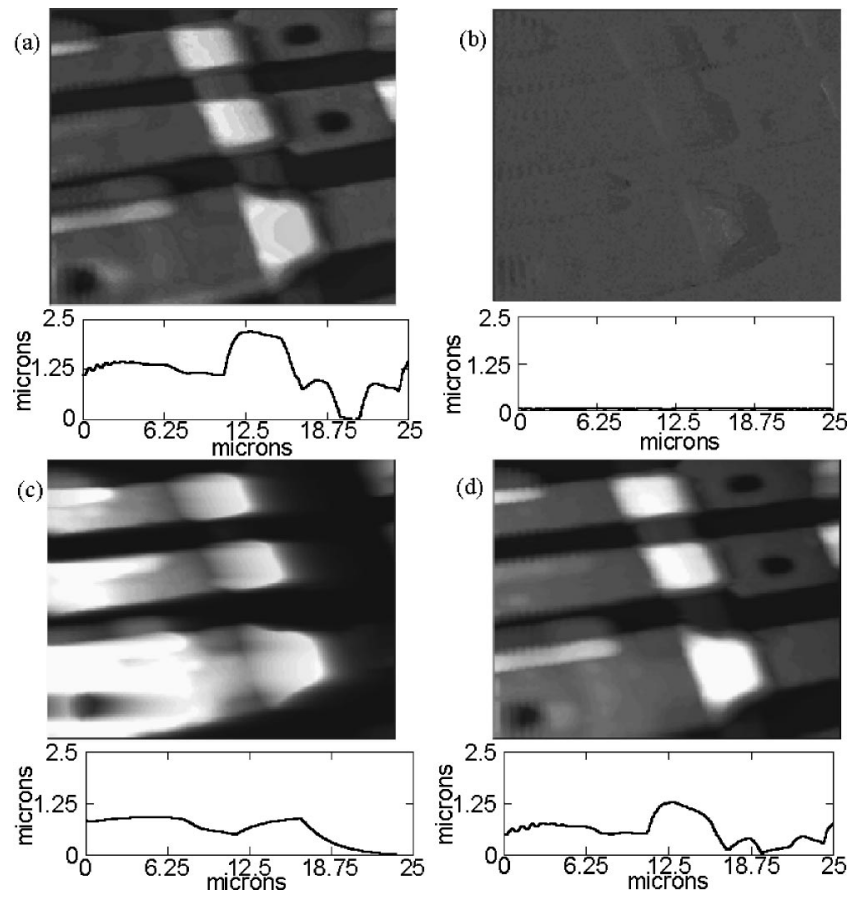

FIG. 4. (a) A $512 \times 512$ pixel, constant force image acquired with a $2 \mathrm{~mm} / \mathrm{s}$ tip velocity and dual integrated actuators. A line profile is shown along the dotted line. (b) shows the composite error signal for the image. (c) and (d) show the thermal actuator and the $\mathrm{ZnO}$ actuator signals, respectively. The sample is an integrated circuit with features over 2 microns in height.

sharp change in power from the quadratic dependence on applied voltage. The $p-n$ junction after breakdown acts as a diode and clamps the voltage across the heater. A resistor placed in series with the thermal actuator, will limit the current in this breakdown region. In this regime, the power dissipated by the heater becomes proportional to the resistor controlled current. Figures 3(a) and 3(b) show the power dissipation through the heater (obtained by measuring current and applied voltage). In Fig. 3(a), a $3 \mathrm{k} \Omega$ resistor was used in series with the thermal heater, thus showing a linear region with finite slope after breakdown. Figure 3(b) shows this effect with a $30 \Omega$ resistor in series with the thermal heater. After breakdown, the current increases rapidly, and so therefore does the power. Operating in the breakdown region is an effective way to linearize the response of the thermal actuator. Moreover, the deflection sensitivity of the actuator can be tailored from an arbitrarily small sensitivity to a pseudobistable state simply by adjusting the series resistor.

The measured deflection curve for the thermal actuator with applied voltage below breakdown is shown in Fig. 3(c). The quadratic voltage response of the cantilever causes the cantilever deflection to be symmetric around zero bias. Therefore, a dc offset must be applied to the thermal actuator for operation $(6 \mathrm{~V})$ so that voltage never drops below zero. Likewise, because the deflection response is quadratic, signal fluctuations must be kept small compared to the offset bias such that displacement can be considered linear in the feedback regime. Alternatively, an integrated circuit which outputs the square root of the input can be used before the thermal actuator to linearize the response. Figure 3(d) shows the linear response of the thermal actuator in series with the
$3 \mathrm{k} \Omega$ resistor, when operated in the breakdown region.

In Fig. 4(a), we show a high speed constant force image with the dual actuators operating in the nested feedback loop. Figures 4(c) and 4(d) show the thermal and $\mathrm{ZnO}$ actuator signals, respectively. Figure 4(b) is the composite error signal. The image was taken with a tip velocity of $2 \mathrm{~mm} / \mathrm{s}$ by raster scanning at $40 \mathrm{~Hz}$ over a $25 \mu \mathrm{m}$ by $25 \mu \mathrm{m}$ area. The sample came from an integrated circuit chip. Visible are metal lines and contact holes containing features over $2 \mu \mathrm{m}$ in height. In this work, the voltage applied to the $\mathrm{ZnO}$ was limited to $\pm 15 \mathrm{~V}$. With this voltage range, the $\mathrm{ZnO}$ actuator alone is not able to deflect over the entire $2 \mu \mathrm{m}$ range of the sample, so the use of the thermal actuator increases the effective range of the high speed actuator. This 512 $\times 512$ pixel image was acquired in under $13 \mathrm{~s}$. The ringing observed in the image is due to the $x-y$ piezotube scanner and not the $\mathrm{ZnO}$ actuator.

We find that neither the signal to the piezoelectric actuator nor the signal to the thermal actuator completely contain all the image topography [Figs. 4(c) and 4(d)]. The two signals must be appropriately combined to record the topography as shown in Fig. 4(a).

In conclusion, we present a flexible system for high speed imaging over extended ranges. The feedback gain of the thermal actuator can be adjusted to either acquire the low frequency portions of the image data, or to simply remove imaging artifacts such as sample slope. We have demonstrated that for single tip operation, the thermal actuator can be replaced with the standard piezotube. However, for applications involving arrays of cantilevers, ${ }^{11}$ the second actuator must be integrated, which is the natural environment for combining the high speed actuator with the thermal actuator.

The authors would like to thank Scott Manalis at MITs Media Lab and Gene Franklin for their professional expertise and Jim Zesch at Xerox PARC for the $\mathrm{ZnO}$ films. This work was supported in part by DARPA, ONR, and NSF. J.D.A. and D.A.F. acknowledge the support of a NSF graduate fellowship.

${ }^{1}$ S. Akamine, T. R. Albrecht, M. J. Zdeblick, and C. F. Quate, IEEE Electron Device Lett. 10, 490 (1989).

${ }^{2}$ T. Itoh and T. Suga, in The Proceedings of Transducers '95, Stockholm (Royal Swedish Academy of Engineering Science, 1995), Vol. IV A, p. 632.

${ }^{3}$ T. Fujii, S. Watanabe, M. Suzuki, and T. Fujiu, J. Vac. Sci. Technol. B 13, 1119 (1995).

${ }^{4}$ S. R. Manalis, S. C. Minne, and C. F. Quate, Appl. Phys. Lett. 68, 871 (1996).

${ }^{5}$ S. C. Minne, G. Yaralioglu, S. R. Manalis, J. D. Adams, J. Zesch, A. Atalar, and C. F. Quate, Appl. Phys. Lett. 72, 2340 (1998).

${ }^{6}$ S. R. Manalis, S. C. Minne, A. Atalar, and C. F. Quate, Rev. Sci. Instrum. 67, 3294 (1996).

J. G. Smits and W. S. Choi, Proceedings of IEEE 48th Annual Symposium on Frequency Control, Boston 1994 (unpublished), p. 139.

${ }^{8}$ S. R. Manalis, S. C. Minne, A. Atalar, and C. F. Quate, Appl. Phys. Lett. 70, 3311 (1996).

${ }^{9}$ S. C. Minne, G. Yaralioglu, S. R. Manalis, J. D. Adams, J. Zesch, A. Atalar, and C. F. Quate, Appl. Phys. Lett. 72, 2340 (1998).

${ }^{10}$ J. R. Barnes, R. J. Stephenson, C. N. Woodburn, S. J. O'Shea, M. E. Welland, T. Rayment, J. K. Gimzewski, and Ch. Gerber, Rev. Sci. Instrum. 65, 3793 (1994).

${ }^{11}$ S. C. Minne, P. Flueckiger, H. T. Soh, and C. F. Quate, J. Vac. Sci. Technol. B 13, 1380 (1995). 